\title{
Parenting the Premature Infant: Potential Iatrogenesis from the Neonatal Intensive Care Experience
}

Joan M. Rikli, RN, MS

Volume 3
Indexing terms: infant, premature; intensive care units, neonatal; parenting; parent-infant relations; outcomes of prematurity; professional-family relations; family-centered care; role; parents; psychosocial aspects of illness

\section{Abstract}

(1) Developmental outcomes of premature infants are associated with the quality of the home environment and the level of parenting skills the family possesses. Successful development of the parenting role may be negatively influenced by the Neonatal Intensive Care Unit (NICU) environment and nursing practices. Identification of interventions that promote the development of parenting skills in the NICU can potentially improve developmental outcomes for premature infants.

\section{Statement of the Practice Problem}

(2) The purpose of this paper is to identify aspects of the neonatal intensive care (NICU) experience that negatively impact upon the parents' ability to successfully parent their infant after discharge. It has been identified that the NICU experience can lead to disturbances in parenting (Gennaro, 1991 [5]). Gennaro states, "These disturbances may be as minor as increased clumsiness and uncertainty in caretaking activities or as major as child abuse" (p.55 [5]). It is also known that the home environment and the level of parenting skills the family possesses contributes significantly to the eventual developmental outcome of the premature infant (Schraeder, Rappaport, \& Courtwright, 1987 [18]). In some cases, the disturbances in parenting that occur due to the NICU experience may contribute to a poorer developmental outcome for the infant. Therefore, identification of factors that impact the parents' abilities to successfully parent their premature infants can lead to interventions that minimize negative effects of the NICU experience, strengthen parenting skills, and improve the long-term outcome of the infant.

(3) Low birth weight and premature delivery continue to be major problems in the United States. Ladden (1990 [9]) reported that in the United States, $7 \%$ of all infants were considered low birth weight (less than $2500 \mathrm{gm}$ ). Nineteen percent of these infants were rehospitalized in the first year of life, as opposed to $8 \%$ of normal birth weight infants. The costs to society, both economically in health care utilization dollars and socially in terms of lower functioning children, are significant.

\section{Summary of the Literature}

(4) This summary is based on searches of MEDLINE and CINAHL from 1975 to 1995 . Search parameters included parentchild relations; parenting: infant, newborn; intensive care unitsneonatal; intensive care, neonatal; and parents. A description of research articles included in this summary can be found in Table 1.

(5) In order to identify factors in the NICU experience that impact parents' abilities to successfully parent their premature infants it is important to identify determinants of successful parenting in general, and also describe the characteristics of the premature infant and tasks of parenting that enter into the equation. With this information, the NICU experience can then be explored in context.

\section{Tasks and Characteristics of Successful Parenting}

(6) Belsky (1984 [1]) describes three determinants of parental functioning. These include the parents' personalities and psychological well-being, contextual subsystems of support, and the child's temperament. Contextual subsystems of support encompass emotional support, advice and information, and the provision of social expectations (designation of what is and is not appropriate behavior). He stresses that "parenting that is sensitively attuned to children's capabilities and to the developmental tasks they face promote a variety of highly valued developmental outcomes, including emotional security, behavioral independence, social competence, and intellectual achievement" (p.85 [1]). McCain (1990 [10]) identifies tasks that parents and infants must accomplish in order to achieve optimal developmental outcome of the infant. The infants' tasks include demonstrating their needs through cues (crying, fussing, sleeping, eating, etc.) and responding to the parent (smiling, making eye contact, vocalizing, etc.). The parents' tasks include recognizing the infant's cues and responding to them appropriately. This implies a reciprocity between the parent and infant, where each gives feedback to the other.

(7) Mintzer, Als, Tronick, and Brazelton (as cited in Yoos, 1989 [19]) identified that in preterm infants, reciprocity of feedback may not be present to the same extent as in interactions with full term infants. Preterm infants are less attentive and responsive than full term infants. They are more irritable, disorganized, difficult to feed, and their cues are harder to 
interpret. They give less positive feedback to parents behaviorally. They may not like to be held close, will spit and squirm while being fed, and rarely make eye contact or smile. Therefore, premature infants may be inherently more difficult to parent.

\section{Long-Term Outcomes Related to Parenting Difficulties}

(8) Gennaro (1991 [5]) reports that confidence in parenting abilities influences the use of health care services once home. She has found that mothers of low birth weight infants who have less confidence in their parenting skills demonstrate a higher rate of acute care visits than those with more confidence. These infants were not sicker or smaller than other infants during their hospitalization, but their mothers had higher levels of depression and anxiety.

(9) Parents of premature infants may feel less confident about their parenting abilities than parents of full term infants (McCain, 1990 [10]; Zahr, 1991 [20]). Zahr reported a relationship between confidence levels and infant temperament. Mothers who were confident in their parenting roles rated their infants' temperaments as less difficult. Although causality was not proven, it was suggested that perhaps those infants who had difficult temperaments negatively influenced their mother's confidence in their ability to care for them. It was proposed that increased maternal confidence levels, obtained through the provision of support and information about the premature infant's temperament, may assist the mother in caring effectively for her baby and adapt to the parenting role successfully.

(10) Schraeder (1986 [17]) found that $67 \%$ of the variance in developmental outcome of very low birth weight infants (less than $1500 \mathrm{gm}$ at birth) seen at one year of age can be attributed to the home environment. She suggested that the infant influences and reinforces parental responses, and the parents' behaviors and characteristics support developmental gains. In a follow up study, she indicated that parents who were involved with their infant and engaged in appropriate developmental activities (play and language stimulation) contributed to better developmental outcomes for their infant (Schraeder, Rappaport, \& Courtwright, 1987 [18]).

\section{Impact of the NICU Environment}

(11) The Neonatal Intensive Care Unit (NICU) can be a very intimidating place for parents. It is filled with "high tech" equipment, multitudes of health care workers, and tiny, fragile infants. Faced with such an environment, parents often feel misplaced, lost, and frightened (Pinch \& Spielman, 1990 [15]). As a result, they may take on the role of bystander, rather than becoming an active participant in their child's care (Kenner, 1990 [7]). They often do not provide care to their infant until shortly before discharge. The parents' minimal participation in caregiving activities while their premature infant is in the NICU can lead to a lack of confidence in parenting skills and ineffective problem-solving regarding the infant's care once home.

(12) While the child is hospitalized in the NICU, parents may not feel that the infant is theirs, but instead belongs to the nurses and doctors (Norris \& Hoyer, 1993 [14]). Parents do not take on the parenting role and thus feel a lack of responsibility for the infant's care. Norris and Hoyer inferred from the literature that this disengagement of parents is due to the staff's focus upon the technical, medical aspects of the infant's condition, with a lack of attention to the psychosocial needs of the infant and family, and the failure of staff to encourage parental involvement. They describe a discrepancy between the rights and responsibilities of parents as perceived by nurses and parents. These discrepancies occur in the areas of degree of control exercised, the role of the parent in caregiving activities and the provision of information. Control clearly lies in the hands of the staff and is conferred upon parents at the discretion of the nurse. While some parents have no desire for greater control, input, or participation, others need greater involvement in order to take on the parenting role.

(13) Miles (1989 [11]) outlined aspects of care that contribute to the parents' stress levels. These include: the infant's frail appearance, feelings of helplessness in not knowing how to comfort the infant and protect it from pain, separation from the infant due to distance from the hospital or family responsibilities at home, and not being able to hold the infant or participate in his or her care to the degree desired. She further stated that stress engendered in the NICU may negatively influence parenting behaviors (Miles, Funk, \& Carlson, 1993 [12]).

(14) Many parents feel overwhelmed by the prospect of being totally responsible for their infant's care after discharge. Kenner (1990 [7]) found that this fear of responsibility is related to the lack of practice in the parenting role that families experience in the NICU environment. When surveyed, parents stated they felt devalued in the NICU (Kenner, 1990 [7]). They were unable or not allowed to participate in the care of their infant. Their role was not clearly communicated to them. As a result, some parents experienced difficulty making independent decisions about their child's care once home. She describes parents calling the physician after discharge for permission to change from disposable diapers to cloth, illustrating their inability to exercise basic problem-solving skills. In a survey conducted of parents after discharge, Kenner and Lott (1990 [8]) found that many experience feelings of low self-esteem, as well as failure and inability to cope. These parents felt they did not really become parents until the baby came home and they had total care responsibility. However, once home, the parents questioned their ability to care for their infant. The lack of positive reinforcement from the infant caused the parents to doubt their caregiving abilities. They believed the nurses in the NICU were able to handle the infant's temperament and recognized what the infant needed, and found themselves lacking in comparison.

(15) Some parents feel surprised and unprepared when informed that the infant can go home soon. In spite of the improvement in the infant's medical status, the parent is still concerned for the infant's survival, and therefore unable to attend emotionally and intellectually to discharge teaching (Kenner, 1990 [7]). The "Care-by-Parent Unit" described by Consolvo (1986 [2]) attempted to decrease anxiety prior to discharge by providing a safe environment for the parents to independently practice caregiving activities while their baby was still in the hospital. Immediate access to nursing staff for advice or assistance was available, if needed. Consolvo found that parents who cared for their infant in the care-by-parent unit experienced less anxiety after participation in the program than prior to the 
program. However, she did not compare these parents' anxiety levels with those of parents who did not participate in the careby-parent unit. Although the interventions proposed in Consolvo's study intuitively make sense, it is difficult to generalize her findings to other settings or populations and determine if her interventions increase confidence levels or decrease anxiety about caregiving abilities once the parent is home.

(16) Several authors discussed the unrealistic expectations that parents may have of their premature infant (Dobbins, Bohlig, \& Sutphen, 1994 [3]; Frodi, Lamb, Leavitt, Donovan, Neff, \& Sherry and Stern \& Hildebrandt as cited in Yoos, 1989 [19]; Kenner \& Lott, 1990 [8]; Miles, 1989 [11]). Preconceived notions of the "ideal" baby are generally based on the appearance and behavior of full term infants. Parents may not have been given information about the behavioral and physical characteristics of premature infants while in the NICU. Strategies for feeding, stimulating, and interacting with premature infants may not have been shared, thus parents are unprepared for the lack of reciprocity they receive from their premature infant and may attribute their infant's state to "not liking the parent." This in turn may lead to a decrease in the effort the parent employs to interact with the infant (Minde, Whitelaw, Brown, \& Fitzhardinge, 1983 [13]). However, parents may choose to limit interaction and stimulation of the infant because they recognize the disorganized nature of its behavioral state. This alternative explanation for the limited interaction noted tween the parent-infant dyads studied by Minde et al., (1980 [13]) should also be entertained.

(17) Seventy-three percent of parents surveyed by Dobbins, Bohlig, and Sutphen (1994 [3]) indicated that they desired to talk with another parent who had survived an NICU experience similar to theirs. Friends and relatives were not considered helpful in putting the premature birth of their child into perspective. These "support people" had little knowledge about the stress parents were under and were unable to provide needed advice or help. A lack of a frame of reference for the "normal" NICU experience hampered the parents' ability to identify appropriate role attributes for parenting in their current situation.

(18) The nurse is potentially one of the biggest barriers to parenting in the NICU. If abused, the nurse's position as gatekeeper to the infant and his or her ability to control and orchestrate the parents' level of involvement can negatively impact the parents' relationship with the infant. Griffin (1990 [6]) identified several areas where the nurses' attitudes toward parents obstructed the development of their parenting role. Parents identified that they were willing to assume greater responsibility for caregiving of their infant than the nurses were willing to allow. However, some nurses viewed parents as a source of stress and frustration and a consumer of their valuable time. They attempted to place the family in one of three roles. The preferred role was that of "visitor." In this role, the parents were expected to be polite, stay for short periods of time, and make few demands of the nurse. Also acceptable was the "worker" role, where the parents assumed some nurturing tasks for the infant. These activities were tolerated as long as they decreased the nurse's workload and the parents' participation was controlled by the nurse. If the parents overstepped these boundaries and attempted to exercise control or make demands, they fell into the "problem" role, and required "dealing with" to get them in line. Parents treated in such a manner responded with anger and frustration, or more commonly became passive, disconnected, and uninvolved in decision-making or caregiving.

(19) Pinch and Spielman (1990 [15]) describe this passive parental behavior as the "medicalization of parenting." Medicalization of parenting occurs when parents learn to accept the priorities and values of the health care providers and focus on the "numbers" and equipment rather than their relationship with the infant. The end result is a parent who does not feel responsible for the infant. When the nurse finally attempts to engage the family as discharge nears, the parents' uncertainty, lack of knowledge, and unfamiliarity with caregiving interferes with their ability to learn the skills necessary to care for the infant at home.

(20) Scharer and Brooks (1994 [16]) conducted a study that examined the relationship between primary nurses and mothers of premature infants. They described how this relationship impacts the mother's ability to parent her infant during hospitalization. The relationship can be difficult, with a struggle for control due to the nurse's discomfort with sharing power. The nurse may not recognize the cues the parents give as to their desires or needs to participate in care delivery. In addition, the parents may be reluctant to share their desires or criticize the actions of the nurse for fear of retribution while their infant is in the hospital. Those parents who became assertive and demanded increased participation and decision-making control were viewed by the health care providers as interfering with care. In addition, some nurses chose to ignore the parents' expressed wishes about the baby, such as how to dress the infant, or to save a feeding for the parents when they visit. Competitive/conflictual relationships developed at times, especially when the nurse was very attached to the infant, or when the nurse questioned the parents' competence to care for the infant. This led to role confusion in the parents, left them wondering who the baby belonged to, and interfered with their assumption of the parenting role.

\section{Annotated Critical References}

(21) Griffin, T. (1990 [6]). Nurse barriers to parenting in the special care nursery. Journal of Perinatal Neonatal Nursing, $4(2), 56-67$.

(22) This article identified types of obstacles to parenting encountered in the special care nursery. The principal barrier identified was the nurse. Other barriers identified were physical (distance from unit, space, unit accommodations), mechanical (equipment or treatments utilized with the infant), and psychological (infant appearance, lack of privacy, feelings of hopelessness, fear of attachment). Nurse barriers resulted from a lack of sensitivity to the needs of the parent or inability to reduce other barriers to parenting. Nurses' attitudes about parental participation in care conflicted with the parents' desire to provide care to their infant. Some nurses feared parents would interfere with the child's treatment. Other nurses enjoyed acting as surrogate parent and were in competition with parents for the infant's affection. The preferred role for parents was that of visitor. Nurturing tasks performed by parents were also 
encouraged, but further participation, unless conferred upon the parents by the nurse, were seen as problematic. Such behavior of nurses was attributed to the value placed on technical aspects of care by staff, and limited life experiences of the nurses, rendering them unable to relate to the needs and desires of parents. Strategies for reducing nurse barriers to parenting included review of policies and procedures that apply to parents and identification and correction of inappropriate nurse behaviors. The use of a parent-staff agreement form was proposed to document care practices that parents may perform. This "contract" represented a negotiation point and legitimized the partnership of the parents in caregiving. Educating nurses about the needs of parents of premature infants, and issues regarding family-nurse relationships was also suggested. Recognition by staff that the infant belongs to the parents and acceptance of the obligation to include parents in caregiving throughout the hospitalization were identified as essential. As this article draws its conclusions and recommendations from observations of the author and a review of the literature, rather than from research, care must be taken when applying its findings to other special care nurseries.

(23) Miles, M.S. (1989 [11]). Parents of critically ill premature infants: Sources of stress. Critical Care Nursing Quarterly, 12(3), 69-74.

(24) The purpose of this study was to identify environmental stimuli that parents found stressful during their infant's stay in the NICU. The most stressful items, as measured on the Parental Stress Scale: NICU, consisted of the child's appearance and behavior and parental role alteration. The fragile, sick appearance of the infant caused the parents the most stress, followed by their distress at being separated from and not knowing how to help their infant. Additional highly stressful items included not being able to hold the infant, being unable to protect the infant from pain, and not being able to care for the infant. The author suggested interventions with parents that help them understand and cope with their infant's appearance and behavior, assist them in reducing separation from the infant, and enable them to practice the parental role in the NICU.

(25) Kenner, C. \& Lott, J.W. (1990 [8]). Parent transition after discharge from the NICU. Neonatal Network, 9(2), 31-37.

(26) This article describes a descriptive study in which parents of NICU graduates identified their experiences upon transition from the NICU environment to the home. Parent responses to discharge included feelings of low self-esteem, failure, and inability to cope. The prospect of taking on total responsibility for the care of their premature infant initiated these fears and feelings. The lack of positive interaction and feedback from the infant caused parents to question their ability to properly care for the baby. Lack of caregiving experience while in the NICU contributed to the parents' lack of confidence in their parenting skills. Parents identified five areas of concern that impacted on their ability to feel confident with their infant once discharged. These included informational needs (general infant care and medical diagnosis of the infant), anticipatory grief (persistent fears that the infant might die), parent-child development (characteristics of their infant's behavior and the interaction between the parent and infant), stress and coping (due to the lack of experience in making independent judgments about their infant's needs prior to discharge), and lack of social support. Kenner and Lott recommend several practice changes to address the concerns that parents identified in this study. Staff need to develop flexible discharge tools that meet the individual family's needs as identified by the parents. In addition, follow-up care, by telephone or in person, will increase the parents' perception of social support and support their coping abilities. Parents must be included in the care delivery while in the hospital. Practice in exercising independent judgment regarding the infant and education about the infant's behavioral cues and interaction style are also components of discharge teaching.

(27) Gennaro, S. (1991 [5]). Facilitating parenting of the neonatal intensive care unit graduate. Journal of Perinatal Neonatal Nursing, 4(4), 55-61.

(28) Gennaro reports that there is an increased risk of child abuse among NICU graduates. In this article, she identifies behavioral characteristics of premature infants that contribute to difficulties in parenting. The relationship between the level of parenting skills and the development of the preterm infant is elucidated. The parent's confidence in his or her parenting skills influenced the use of health care services. Mothers experiencing high levels of anxiety utilized a greater number of acute care services for their infants than mothers with lower levels of anxiety, even though their infants were not smaller or sicker during hospitalization. Several interventions for facilitating parenting of premature infants were proposed. The primary intervention noted was to promote parental contact and involvement in the provision of care to the infant while it is hospitalized. Providing emotional support to parents and aiding them in obtaining support from extended families was also suggested. Education about the infant's behavioral cues, and how to organize the infant's behavior were identified as crucial.

(29) Scharer, K. \& Brooks, G. (1994 [16]). Mothers of chronically ill neonates and primary nurses in the NICU: Transfer of care. Neonatal Network, 13(5), 37-47.

(30) The purpose of this study was to determine how the relationship between the primary nurse and the mother of a chronically ill neonate influences the development of the mother's parenting role. Primary nurses and mothers of infants hospitalized in the NICU were interviewed to obtain their perceptions of their relationship with each other, to identify stages that occur in the relationship and to describe how the relationship changes over time to allow the mother to assume more of the care responsibilities for her infant. Four stages in the transfer of care from nurse to mother were identified. These were: (1) nurse providing care, (2) sharing normal infant care, (3) sharing normal infant and technical care, and (4) parent providing care. Factors which negatively influenced the progression of stages included the inability of some nurses to read the mother's cues indicating readiness for participation in care, lack of assertiveness on the mother's part or inability to be clear about her needs, some nurses'concerns about the competence of the mother, variation in the nurse's willingness to respect the parents' wishes about the infant's care, and competition or conflict in the relationship between the nurse and mother. The authors recommend that nurses carefully examine their relationships with parents to be 


\section{Table 1: Studies Regarding the Parenting of Premature Infants}

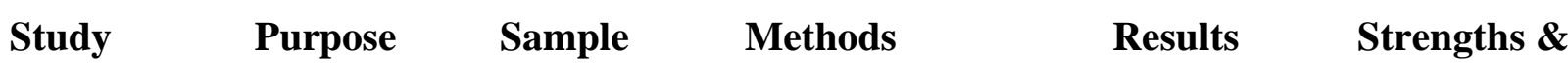 \\ Limitations} (1986 [2])

Relieving parental anxiety in the care-byparent unit.

Dobbins, N., Bohlig, C., \& Sutphen, J. (1994 [3])

Partners in growth:

Implementing family-centered changes in the neonatal intensive care unit.

Gennaro, S. (1986 [4]) Anxiety and problemsolving ability in mothers of premature infants. maternal anxiety is decreased when mothers have 24 hour total care responsibilities prior to discharge.

Identification of variables that are perceived by parents to interfere with assumption of the parent role while in the NICU.

McCain, G. (1990 [10]) Parenting growing preterm infants.
Identification Convenience of factors which sample of 40 are anxietyprovoking for mothers of premature infants.

Identification of concerns of parents of premature infants.
Convenience sample of 24 parents of premature infants. NICUs in one
In the first week of hospitalization, mothers completed the Demographic and Obstetrical Data Profile, State-Trait Anxiety Inventory, and the city. All mothers Means-Ends Problemwere married. Solving Procedure.

Anecdotal information provided by parents after discharge from the NICU.
Mothers had less anxiety after being in the care-byparent unit than before participation.
Not generalizable to other NICUs. The study would have been strengthened if there had been a control group.
Length of stay and distance of home from the hospital had a significant impact on the parents' ability to participate in care. Parents wanted support from other families that had experienced an NICU stay.

Mothers with good problemsolving skills had higher levels of anxiety than those who had lower problemsolving skills.

Positive benefits of anxiety hypothesized.

Parents had concerns in the areas of premie health and development, their parenting abilities the effect of the new baby on family functioning, and the impact on finances.
Only one unit was studied. Results not easily generalized. Strategies were identified that may increase parents' participation in care.

No controls for age or parity. Anxiety levels were not measured prior to admission, so no baseline levels of anxiety were determined. Application of findings to practice is elucidated. 


\section{Table 1 (cont): Studies Regarding the Parenting of Premature Infants}

\begin{tabular}{|c|c|c|c|c|}
\hline Study & Purpose & Sample & Methods & Results \\
\hline
\end{tabular}

Miles, M.

(1989 [11])

Parents of

critically ill

premature

infants:

Sources

of stress.

Minde, K.,

Whitelaw, A., Brown, J., \&

Fitzhardinge,

P. (1983 [13])

Effect of

neonatal

complications

in premature

infants on early

parent-infant

interactions.

Pinch, W. \&

Spielman, M.

(1990 [15])

The parents'

perspective:

Ethical decision-

making in

neonatal

intensive care.

Scharer, K. \&

Brooks, G.

(1994 [16])

Mothers of

chronically

ill neonates

and primary

nurses in the

NICU: Transfer of care.
Identification

of environment-

al stimuli in

the NICU that

are stressful

to parents.

Fifty-three

parents of 37

infants hospital-

ized in two

NICUs. 63\%

were mothers,

$76 \%$ were

white.

To identify

which factors

affect a

premature

infant's long-

term develop-

mental and

behavioral

outcome.

\section{4 premature \\ infants \\ hospitalized \\ in one NICU \\ between 1976 \\ and 1980.}

Examination of

the parents'

perspective

regarding

decision-making

in the NICU.

A convenience sample of 32

families of high risk newborns

from a level

three nursery

were interviewed

prior to the

infant's discharge.

Exploration of

the impact of

the relationship

between the

primary nurse

and mother on

the mother's

ability to parent

Ten mothers

and 9 primary

nurses located

in two NICUs.

Six of ten

mothers and

all nurses were

white. None of

in the NICU. the nurses had

children.
Parents completed the

Parental Stress Scale:

NICU and a personal-

situational survey.

Observation of infants'

behavioral state and

mothers' interactions

with their infants while

in the hospital and after

discharge. Infants rated

on a morbidity scale.

Phenomenological approach was used

to describe percep-

tions of families

regarding ethical

decision-making.

Grounded theory

methodology,

qualitative approach.

Two semistructured

interview guides,

one for nurses, one

for parents.
The most

stressful items

were the infant's

appearance and

the parental

role alteration.

The sickest infants showed a reduction in motor activity and their mothers interacted with them less as compared to mothers of less sick infants.

Parents adopted a passive role in decision-making. Most families felt disconnected from their infant, and had limited interactions with their infant.

Transfer of care from the nurse to the mother occurred gradually and moved through
Interviews occurred prior to discharge, when the viability of the infant was not yet determined.

Families from only one

NICU were interviewed.

Testing to assure the trustworthiness and credibility of the research was detailed. The fact that the majority of the nurses in the sample The pa
sample
tative
white.
arge, a
res, ationt
ore not
of the
at reci

The impact of social class, physician attitudes, and the ation of her addressed. One of the first studies to look at reciprocity infant and parent.

The tool utilized had been tested for content validity population at large, as the majority were white. four fluid stages. influenced their responsive-

Nurse providing ness to the mothers.

care, sharing

normal infant

care, sharing

normal and

technical care,

and parent

providing care. ness to the mothers.

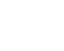




\section{Table 1 (cont): Studies Regarding the Parenting of Premature Infants}

\begin{tabular}{|c|c|c|}
\hline Study & Purpose & ample \\
\hline
\end{tabular}

Schraeder, B. Investigation Forty-one The HOME Inventory

(1986 [17]) of the medical, VLBW infants was utilized during a

Developmental biological, and and their home visit when the

progress in

very low

birth weight

infants

during the

first year

of life.
12 months old. Other

tools used were the

MCDI and DDST.

\section{environmental mothers.}

influences on Weights of the

developmental infants ranged

outcome of from $780 \mathrm{gm}$

the very low to $1500 \mathrm{gm}$.

birth weight

(VLBW)

infant in the

first year of life.
Schraeder, B.,

Rappaport, J.,

\& Courtwright,

L. (1987 [18])

Preschool

of very low

birthweight

infants. development

\section{Description of \\ the influence \\ Forty VLBW infants were perinatal insults, examined at neonatal illness, home visits at and environment $6,12,24$, and have on the 36 months of cognitive development of VLBW infants during the first three years of life. age (corrected).}

Zahr, L. (1991 [20]) Investigating the relationship

The relationship between the between maternal confidence and mother-infant behaviors in premature infants.

\section{mother's} confidence in her parenting abilities and the infant's temperament. mothers and premature infants from low socioeconomic status. infants were six and
The HOME Inventory for preschool children was used to assess cognitive, emotional, and social support provided to the subjects. The children were also tested with the MCDI.
There is a causal relationship between the quality of the home environment and developmental outcomes at one year of age.

Premature infants may also have a natural "self-righting" tendency.

Forty-nine percent of the variance in development seen at 36 months was attributed to environmental factors. Most significant were the parent's stimulation of language and academic behavior.

Forty-nine Maternal behaviors were assessed in the NICU, and at 4 and 8 months after discharge. Infant temperament was assessed with the Bates Infant Characteristic Questionnaire.
Confidence was not related to severity of neonatal illness, but was related to educational level, income, and previous parenting experience. Confident mothers rated their infant's temperament as less difficult than mothers with less confidence
The importance of long-term support and follow-up for families is emphasized. Results were from only one NICU, so findings should be generalized with caution.

The need for a larger sample and control group was identified by the authors. The importance of community resources available after discharge is identified.

Causality between confidence levels and rating of infant temperament was not determined. Measures to increase maternal confidence were suggested. 
sure they are reading the parents' cues correctly. They should strive to decrease competition and conflict between themselves and parents. In addition, nurses and parents should work together to smoothly transfer care responsibility to the parent as the infant improves.

\section{Practice Implications}

(31) Interventions to promote positive parenting skill development of parents of premature infants can be categorized by the determinants of parental functioning as described by Belsky (1984 [1]). These include the parents' personalities and psychological well-being, contextual subsystems of support, and the child's temperament. Contextual subsystems of support encompass emotional support, advice and information, and the provision of social expectations (designation of what is and is not appropriate behavior).

\section{Interventions that Support the Parents' Personality and Psychological Well-being}

(32) It is difficult to impact the parent's personality, but interventions that affect psychological well-being have been identified. Gennaro (1986 [4]) studied the maternal anxiety levels of women of infants in the highest, high, or median neonatal risk categories. There was no significant difference in the anxiety levels between the three groups. However, mothers with higher problem-solving abilities demonstrated higher levels of anxiety than those with lower levels of problem-solving abilities. Rather than attempting to diminish anxiety for these women, Gennaro suggests nurses reassure mothers that anxiety is a normal reaction to the NICU experience and help them to channel this anxiety into activities that help her prepare for the infant's discharge.

(33) Kenner (1990 [7]) recommends that nurses encourage parents to express their negative and positive feelings about the NICU experience. Parents often feel angry that their infant is ill, and feel guilty that they have not been able to prevent the premature birth. Open communication of their fears may contribute to their psychological well-being.

\section{Contextual Subsystems of Support}

\section{Emotional support}

(34) Gennaro (1991 [5]) identified the provision of emotional support to parents as a critical part of the neonatal nurse's role. In addition, nurses need to assist parents in identifying external supports available to them, such as grandparents, friends, or other relatives. Scharer and Brooks (1994 [16]) emphasized the need for nurses to recognize cues that parents give indicating their desire to participate in care. Whenever possible, the parents' wishes regarding care practices should be respected (such as the use of pacifiers, how to dress the infant, saving feedings for parents when requested). In recognition of the impact of the infant's appearance on the parents, Miles (1989 [11]) suggests interventions to prepare parents for what they will see, and recommends covering up bruises, wires, etc. whenever possible.
Normalizing the infant's appearance by placing a diaper and clothing on the child as soon as possible also decreases the stress caused by the infant's frail demeanor. Restricting visitors to parents and grandparents denies families of needed emotional support. Self definition of family members by parents enables them to have those individuals deemed most supportive available to them when visiting their infant (Griffin, 1990 [6]).

\section{Instrumental assistance}

(35) Instrumental assistance includes advice-giving, instruction, and help in performing technical tasks. The nurse's role throughout hospitalization is to aid in the infant's assimilation into the family (Griffin, 1990 [6]). This requires the family to be able to function as capable caregivers for their infant. Ongoing opportunities for parental involvement in care activities in every stage of the infant's illness is necessary for the parents to gain a level of confidence in their parenting skills (Kenner,1990 [7]). Practicing decision-making and provision of care in the safe atmosphere of the NICU allows parents to ask questions and obtain immediate assistance if needed, but gives them an opportunity for independence as well. It is important for nurses to base discharge teaching on areas identified by parents rather than relying on standard care plans or material the health care provider has determined necessary. Telephone follow-up once home also offers the family a "safety net" where questions can be safely asked of staff who are familiar with their infant's needs.

\section{Social expectations}

(36) It is important for parents of premature infants to determined the components of the parenting role that pertain to their particular situation. Parent groups and contact with "veteran NICU parents" help validate feelings and concerns about parenting an infant in the NICU and once home. Contact with other parents of preterm infants was identified as an effective way to increase feelings of competence (Minde et al., 1983 [13]).

(37) Many parents have expressed confusion over their role in the NICU. Frank discussion and explanation of the transfer of care from nurse to parents is a mutual responsibility of nurses and parents (Scharer \& Brooks, 1994 [16]). Nurses also need to guard against "medicalization of parenting," where parents learn to focus on numbers and equipment rather than their relationshipwith the infant (Pinch \& Spielman, 1990 [15]). As this leads to disengagement of the parent from responsibility for the infant, it is important to involve parents in decision-making and the care of the infant, and to share observations of the infant's unique characteristics at each contact with the family. However, Pinch and Spielman also noted that some parents do not desire greater involvement in decision-making for their infant while hospitalized. It is important for the nurse to assess the parents' desires and individualize the plan for meeting them.

\section{Infant Temperament}

(38) The immature neurological system of the premature infant contributes to the behavioral disorganization commonly seen in the first year of life. As reciprocity of interactions between the infant and parent is crucial in developing a satisfying relationship, parents must learn how their infant differs from a 
full term infant. Recognition of the baby's cues, knowing when to initiate and reduce stimulation, competence in "preemie feeding techniques," and knowledge of how to bring the infant to a quiet alert state are all critical skills for the parent of a premature infant to possess (Yoos, 1989 [19]). It is important for the nurse to point out to parents throughout hospitalization the positive aspects of their infant's behavior and his or her unique characteristics, especially how the infant responds to the parent (Kenner \& Lott, 1990 [8]). Nurses need to develop, implement, and evaluate programs that assist parents in refining their skills in interacting with their infants in an ongoing manner. Programs that focus on the relationship between parents and infants are valuable in promoting optimal developmental outcomes.

\section{Research Veeded}

(39) Ongoing research in the area of parenting the premature infant is critical in order to promote the best outcomes for graduates of NICUs and their parents. Through identification of gaps in the current literature, areas for research may be revealed. Exploration of these gaps may increase the profession's knowledge base, contribute to successful interventions in the NICU and in the community, and positively impact outcomes for premature infants and their families.

(40) It has been identified that there is a perceived discrepancy between the desired level of participation by parents and the willingness of the nurse to facilitate the parents' involvement. However, there has not been a study that compares the parents' beliefs and attitudes regarding desired participation and the nurse's attitudes and beliefs. Such a descriptive study would verify or refute the hypothesis that nurses constitute a major barrier in the development of parenting skills in the NICU. Identification of any significant differences between the two groups would allow for further exploration of why differences occur, provide opportunity for discussion of the appropriateness of attempting to close the gap, and determine the components of programs which would strengthen the parenting experience during hospitalization.

(41) Further investigation into the effect of participation by parents in particular caregiving activities upon the parenting role and the relationship with developmental outcomes is needed. Additional studies could address whether parental decisionmaking in the NICU is a significant issue. Does parental participation in care assist them to cope with their child's illness, or increase the parents' confidence levels after discharge? Is the parents' confidence level an important variable, or is competence in caregiving more significant?

(42) Not all parents desire increased involvement or decision-making control while their infant is hospitalized. The development of tools to identify those whose needs are unique is important, in order to individualize each family's experience. For those parents who do wish to participate to a greater extent, the care-by-parent unit concept described by Consolvo (1986 [2]) may be an effective intervention to increase parent confidence, competence, and parent-infant attachment. Do skills learned in the protected setting of the hospital transfer to the home where immediate support and assistance from experts are not available?
(43) It is not known if high parental anxiety levels upon discharge negatively affect parent-infant attachment. Does anxiety have a beneficial effect on parenting the premature infant as suggested by the association with higher problem-solving abilities reported by Gennaro (1986 [4])? Development of tools to identify which parents are likely to experience high levels of anxiety from the NICU experience is needed.

(44) What are the most effective methods for teaching parents about infant state behavior and temperament? Development of tested protocols and approaches to individualized teaching regarding the differences between premature and full term infants' behavioral responses is needed.

(45) In addition, the components of support and follow-up after discharge in the area of parent-infant interaction has not been identified. Previous programs have focused on growth and development and the medical needs of the infant, rather than addressing ongoing difficulties in parenting a premature infant.

\section{Search Strategies}

\section{Search Terms:}

(46) Parent-child relations; Parenting: Infant, Newborn; Intensive Care Units-Neonatal; Intensive Care, Neonatal; Parents.

\section{Databases Used:}

(47) MEDLINE (1975-1995) English.

(48) CINAHL (1975-1995) English.

\section{References}

1. Belsky, J. (1984). The determinants of parenting: A process model. Child Development, 55(1), 83-96. [MEDLINE Reference]

2. Consolvo, C.A. (1986). Relieving parental anxiety in the care-by-parent unit. Journal of Obstetric, Gynecologic, and Neonatal Nursing, 15(2), 154-159. [MEDLINE Reference]

3. Dobbins, N., Bohlig, C., \& Sutphen, J. (1994). Partners in growth: Implementing family-centered changes in the neonatal intensive care unit. Children's Health Care, 23(2), 115-126.

4. Gennaro, S. (1986). Anxiety and problem-solving ability in mothers of premature infants. Journal of Obstetric, Gynecologic, and Neonatal Nursing, 15(2), 160-164. [MEDLINE Reference]

5. Gennaro, S. (1991). Facilitating parenting of the neonatal intensive care unit graduate. Journal of Perinatal and Neonatal Nursing, 4(4), 55-61. [MEDLINE Reference] 
6. Griffin, T. (1990). Nurse barriers to parenting in the special care nursery. Journal of Perinatal and Neonatal Nursing, 4(2), 56-67. [MEDLINE Reference]

7. Kenner, C. (1990). Caring for the NICU parent. Journal of Perinatal and Neonatal Nursing, 4(3), 78-87.

[MEDLINE Reference]

8. Kenner, C. \& Lott, J.W. (1990). Parent transition after discharge from the NICU. Neonatal Network, 9(2), 31-37. [MEDLINE Reference]

9. Ladden, M. (1990). The impact of preterm birth on the family and society. Part 1: Psychologic sequelae of preterm birth. Pediatric Nursing, 16(5), 515-518. [MEDLINE Reference]

10. McCain, G.C. (1990). Parenting growing preterm infants. Pediatric Nursing, 16(5), 467-470. [MEDLINE Reference]

11. Miles, M.S. (1989). Parents of critically ill premature infants: Sources of stress. Critical Care Nursing Quarterly, 12(3), 69-74. [MEDLINE Reference]

12. Miles, M.S., Funk, S.G., \& Carlson, J. (1993). Parental stressor scale: Neonatal intensive care unit. Nursing Research, 42(3), 148-152. [MEDLINE Reference]

13. Minde, K., Whitelaw, A., Brown, J., \& Fitzhardinge, P. (1983). Effect of neonatal complications in premature infants on early parent-infant interactions. Developmental Medicine and Child Neurology, 25(6), 763-777. [MEDLINE Reference]

14. Norris, D.M. \& Hoyer, P.J. (1993). Dynamism in practice: Parenting within King's framework. Nursing Science Quarterly, 6(2), 79-85. [MEDLINE Reference]

15. Pinch, W.J. \& Spielman, M.L. (1990). The parents' perspective: Ethical decision-making in neonatal intensive care. Journal of Advanced Nursing, 15(6), 712-719. [MEDLINE Reference]

16. Scharer, K. \& Brooks, G. (1994). Mothers of chronically ill neonates and primary nurses in the NICU: Transfer of care. Neonatal Network, 13(5), 37-47. [MEDLINE Reference]

17. Schraeder, B.D. (1986). Developmental progress in very low birth weight infants during the first year of life. Nursing Research, 35(4), 237-242. [MEDLINE Reference]

18. Schraeder, B.D., Rappaport, J., \& Courtwright, L. (1987). Preschool development of very low birthweight infants. Image: Journal of Nursing Scholarship, 19(4), 174-178. [MEDLINE Reference]
19. Yoos, L. (1989). Applying research in practice: Parenting the premature infant. Applied Nursing Research, 2(1), 3034. [MEDLINE Reference]

20. Zahr, L.K. (1991). The relationship between maternal confidence and mother-infant behaviors in premature infants. Research in Nursing and Health, 14(4), 279-286. [MEDLINE Reference]

\section{AUTHOR'S INFORMATION}

Joan M. Rikli, RN, MS, is a Staff Nurse at the Holden Neonatal ICU, University of Michigan Health System. Home address: 2877 Ticknor Ct., Ann Arbor, MI 48104. Telephone: (H) 313-973-6503. Fax: 313-973-0762. E-mail: A598@HOSP.MED.UMICH.EDU 